\title{
Scientific Trends in Clinical Research on Zirconia Dental Implants: A Bibliometric Review
}

\author{
Felice Lorusso ${ }^{1,2}\left(\mathbb{D}\right.$, Sammy Noumbissi ${ }^{1,2} \oplus$, Inchingolo Francesco ${ }^{3}$, Biagio Rapone ${ }^{4}(0$, \\ Ahmad G. A. Khater 5 (D) and Antonio Scarano $1,2, * \mathbb{D}$ \\ 1 Department of Medical, Oral and Biotechnological Sciences, University of Chieti-Pescara, Via dei Vestini, 31, \\ 66100 Chieti, Italy; drlorussofelice@gmail.com (F.L.); sammy@iaoci.com (S.N.) \\ 2 Zirconia Implant Research Group (Z.I.R.G), International Academy of Ceramic Implantology, \\ Silver Spring, MD 20910, USA \\ 3 Department of Interdisciplinary Medicine, University of Bari Aldo Moro, 70121 Bari, Italy; \\ f.inchingolo@icloud.com \\ 4 Department of Basic Medical Sciences, Neurosciences and Sense Organs, University of Bari Aldo Moro, \\ 70121 Bari, Italy; biagiorapone79@gmail.com \\ 5 Faculty of Oral and Dental Medicine, Ahram Canadian University, 6th of October City, 8655 Giza, Egypt; \\ ahmed.g.a.khater@gmail.com \\ * Correspondence: ascarano@unich.it; Tel.: +39-0871-355-4084; Fax: +39-0871-355-4099
}

Received: 18 October 2020; Accepted: 2 December 2020; Published: 4 December 2020

check for updates

\begin{abstract}
Background: The clinical use of zirconia implants has been shown to increase steadily due to their biological, aesthetic, and physical properties; therefore, this bibliometric study aimed to review the clinical research and co-authors in the field of zirconia dental implant rehabilitation. Methods: We searched Scopus and Web of Science databases using a comprehensive search strategy to 5 October 2020, and independently paired reviewers who screened studies, and collected data with inclusion criteria restricted to clinical research only (either prospective or retrospective). Data on article title, co-authors, number of citations received, journal details, publication year, country and institution involved, funding, study design, marginal bone loss, survival rate, failure, follow-up, and the author's bibliometric data were collected and evaluated. Results: A total of 29 clinical studies were published between 2008 and 2020 as 41.4\% were prospective cohort studies and 48.3\% originated from Germany. Most of the included studies had been published in Clinical Oral Implant Research $(n=12)$, and the most productive institution was the Medical Center of University of Freiburg. The author with the largest number of clinical studies on zirconia implants was Kohal R.J. $(n=10)$, followed by Spies B.C. $(n=8)$. Conclusions: This study revealed that zirconia implants have been more prominent in the last ten years, which is a valuable option for oral rehabilitation with marginal bone loss and survival rate comparable to titanium dental implants.
\end{abstract}

Keywords: zirconia implant; bibliometrics; citations; scientometric

\section{Introduction}

The clinical application of dental implant rehabilitation represents consolidated effectiveness in the literature due to long-term predictability and high-level satisfactory functioning and aesthetics [1-4]. Titanium alloys are the most widely used biomaterials for dental implant fixtures due to their physical, chemical, and thermal properties, which produce the osseointegrating ability of the fixture placed to replace the natural teeth [2,5-12].

Recently, the use of zirconia as an implant material has become more prevalent due to its high aesthetic characteristics, particularly in the rehabilitation of the compromised anterior jaw area, where there is fine soft-tissue biotype and the metal sensibility of the patients [13-15]. 
In fact, the literature reports that the titanium ion dissolution related to the implant corrosion could alter the natural oral microbiome and the homeostatic functional balance of the oral tissues [16-20].

On the contrary, it has been shown in vitro that the zirconia surface can lead to a significant decrease in periodontal pathogen adhesion compared to the titanium surface [21], alongside similar bone-implant contact compared to the titanium fixture with an almost overlapping range [22].

Additionally, Scarano et al. reported in a rabbit study that zirconia implants had about $68.4 \%$ bone-implant contact with evidence of contact osteogenesis without fibrous tissue interposition [23].

Zirconia material is distinguished by its clear ivory appearance, which is very similar to the natural color of the teeth and is characterized by an intrinsic strength and physical resistance to the loading [24-26]; as a result, it has been introduced as a restorative material for dental crowns, bars, abutments, and specially designed drills and burs [26-34]. Therefore, zirconia has recently gained further attention in the scientific community by growing research activities to confirm the clinical effectiveness of zirconia as a dental implant material.

Although citations are not an infallible metric to determine whether research is beneficial to researchers and clinicians, citations and citation analysis can quantify an article's influence, author, subject of debate, country, journal, or a specialty $[35,36]$. Based on citation analysis, the bibliometric analysis aims to provide information about the trend in a research field and demonstrates its growth and development [37]; the number of citations received, researcher $\mathrm{H}$-index, and journal impact factor are the most common bibliometric evaluation variables and considered as a scientific productivity score for the scientometric evaluation [38].

With the significant increase in the published articles on dental implants, recognizing trends and advances in a research field is critical and relevant to the needs of dental practitioners and researchers [39,40]. In this sense, bibliometric analysis is a useful tool for this purpose [41,42].

As far as we know, the trends and advances in zirconia dental implants have not been studied before; hence this study aimed to evaluate the bibliometric output of clinical research and co-authors in the field of zirconia dental implant rehabilitations.

\section{Materials and Methods}

We reported this bibliometric study in compliance with the Standards for Reporting Qualitative Research (SRQR) [43] and the Preferred Reporting Items for Systematic Reviews and Meta-Analyses (PRISMA) guidelines [44].

\subsection{Search Strategy}

An online literature search was conducted in Elsevier's Scopus and Clarivate Analytics' Web of Science (WoS) until 5 October 2020. We used the medical terms (MeSH) feature in the Cochrane Library to obtain the available synonyms for our search terms to create a detailed search strategy (Table 1).

Table 1. Search strategy used for each database.

\begin{tabular}{|c|c|}
\hline Scopus & 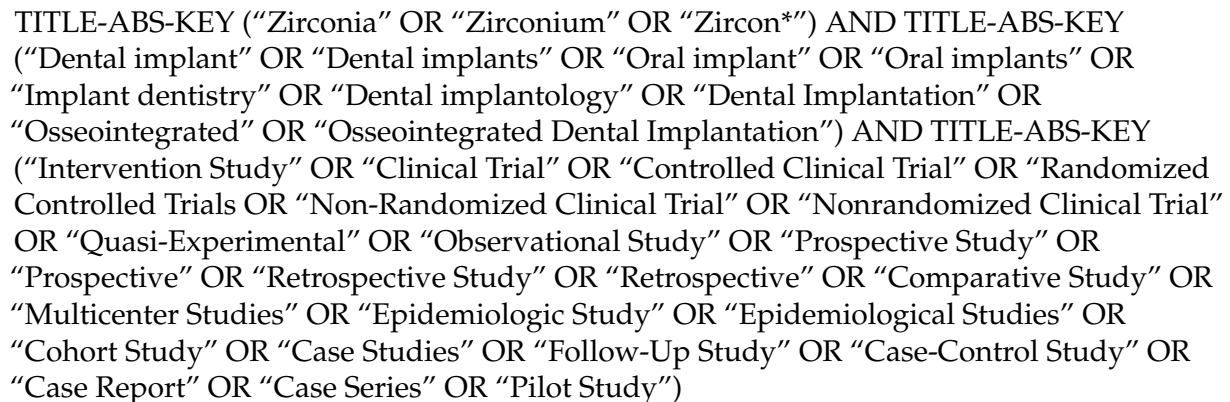 \\
\hline
\end{tabular}


Table 1. Cont.

\begin{tabular}{|c|c|}
\hline Web of Science & 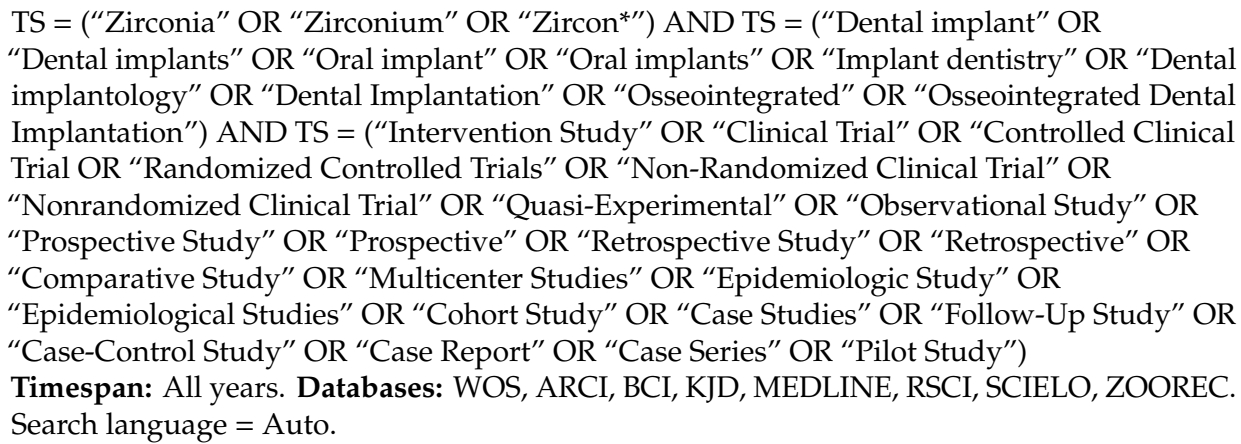 \\
\hline
\end{tabular}

\subsection{Data Extraction and Bibliometric Parameters}

We used a specially built Excel file (Microsoft, Redmond, WA, USA) to collect the findings of the literature search. The file contained the following information: abstracts, year of publication, indexed keywords, journal name, citations as well as all co-author bibliometric data ( $\mathrm{H}$-index, number of papers related to zirconia implant, the total number of papers, citation of paper regarding zirconia implant, and citation of paper regarding zirconia implant). Authors with the highest quantity of clinical studies regarding zirconia dental implants were evaluated and measured the average, the standard deviation, minimum and maximum of topic paper, total papers, topic citations, overall citations, and $\mathrm{H}$-index. Moreover, we evaluated the scientific trend of the included study according to the year of publication and journal details (full title, the impact factor (IF), and rank) based on the Clarivate Analytics report for 2019 with selected categories: "Dentistry, Oral Surgery \& Medicine", study design, number of citations received, marginal bone loss, survival rate, failure, and study follow-up.

\subsection{Study Selection}

We screened the literature search results in two steps, where the first phase was the screening of the title and abstract by paired reviewers separately. Then, the second phase was a full-text assessment by two expert reviewers (L.F and A.S). The reference list of the studies included in the full-text screening was hand-screened for potential additional studies. In this bibliometric study, inclusion criteria were only clinical studies (either prospective or retrospective) without time restrictions. Exclusion criteria were animal studies, in vitro studies, literature reviews, systematic reviews, short communications, personal opinion, letters, book chapters, and non-English studies.

\subsection{Data Analysis}

We used VOSviewer software (version 1.6.8; Leiden University, Leiden, The Netherlands) to visualize a term map analyzing keywords from the data obtained. "Create Map" function was used to analyze the data by using the "Citation" type and setting the unit of analysis as a "number of citations." In the keyword map, the node's size reflects the number of received citations, as the larger size indicates the author with the highest citations. Furthermore, keywords that often appeared together were classified as the same color in network visualization mode $[45,46]$.

\section{Results}

\subsection{Study Selection}

A total of 1159 references were collected from electronic databases in which $(n=185)$ were omitted due to duplication. By title and abstract, 968 articles were screened and 841 excluded as irrelevant topics. By the full-text screening of 127 papers, 29 studies were included in this bibliometric study [47-75] excluding the remaining 98 articles because they did not meet our inclusion criteria (Figure 1). 


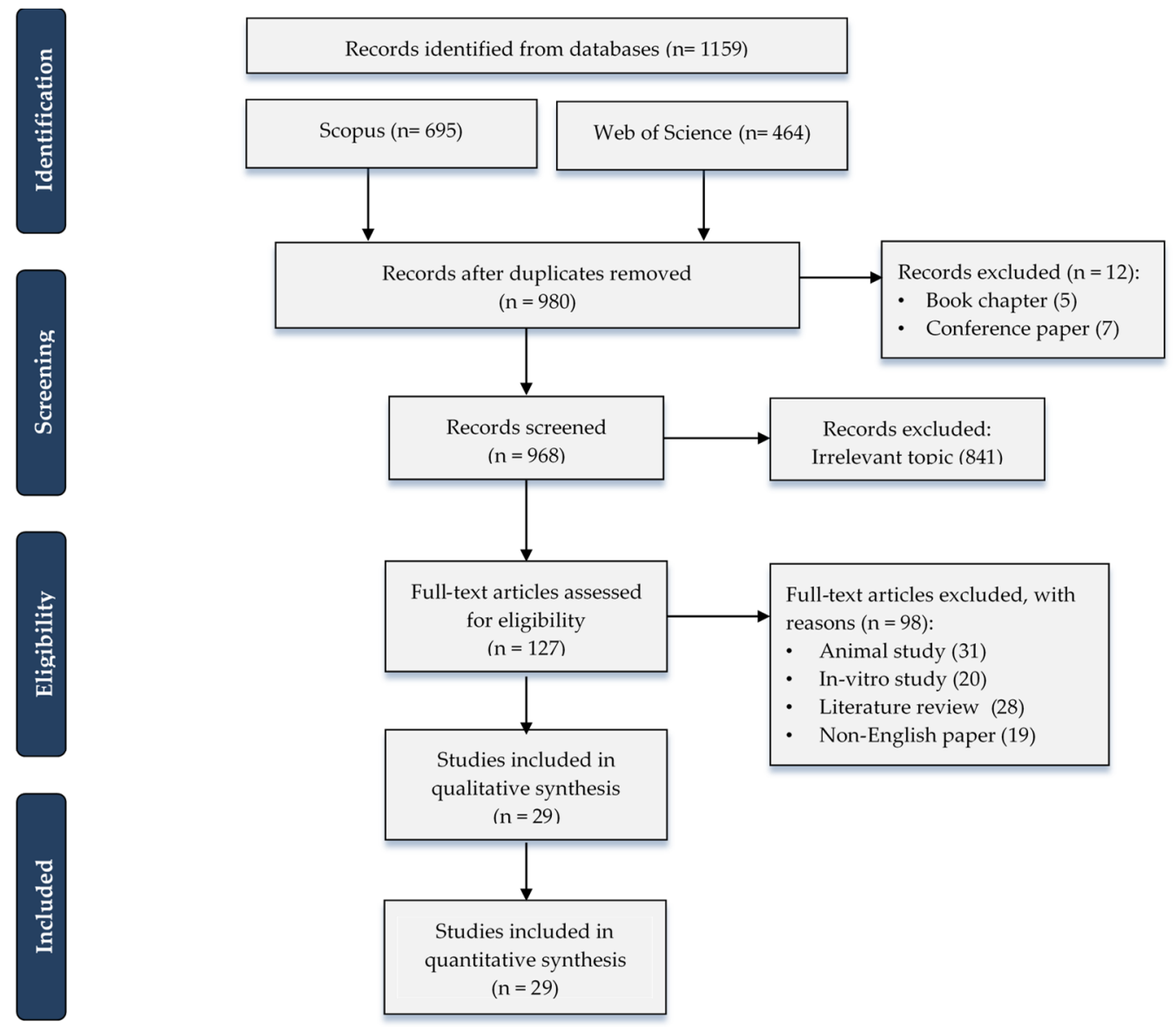

Figure 1. Preferred Reporting Items for Systematic Reviews and Meta-Analyses (PRISMA) flow chart demonstrates the process of literature search and study selection.

\subsection{Study Characteristics}

The included studies showed wide variability in the study design, presence/absence of a control group, experimental site, type of prosthetic rehabilitation, prosthetic connection (one-piece or two-piece), follow-up period, and different methods for evaluating the effectiveness of research. Although these differences exist, most studies reported favorable outcomes for the use of zirconia implants in oral rehabilitation. The main characteristics of the included studies are summarized in Table 2.

A total of 21 studies evaluated monolithic or one-piece zirconia implants $[47-49,51-54,56-58,60$, $62,64,66,70-74,76]$, two of which had titanium implants as their control and showed no significant difference in survival rate and marginal bone loss between groups $(p>0.05)[64,70]$. Two studies evaluated the immediate loading of zirconia implants [53,64]: one study compared it to the non-occlusal loading procedure [64], while the other study compared it with the standard loading protocol [57]. Furthermore, 26 papers assessed the cylindrical microgeometry of zirconia implants [47-65,67,70-75], while three studies evaluated the root-analog zirconia implants obtained by a three-dimensional scan $[66,68,69]$. However, Akça et al. and Pirker et al. reported the lowest marginal bone loss after two years $(0.31 \pm 0.24$ and $0.5 \pm 0.7 \mathrm{~mm}$, respectively), in which Akça et al. used specially designed titanium-zirconia alloy implants [47], and Pirker et al. used specially designed root-analog zirconia implants with a micro-retention surface in a fresh extraction socket [69]. 
Table 2. Main characteristics of the clinical research included (Zir: Zirconia implant group, Tit: Titanium implant group, IF: impact factor, RCT: Randomized controlled trial).

\begin{tabular}{|c|c|c|c|c|c|c|c|c|c|c|c|c|}
\hline \multirow{2}{*}{$\begin{array}{c}\text { Authors } \\
\text { (Year) [Ref] }\end{array}$} & \multicolumn{3}{|c|}{ Journal } & \multirow{2}{*}{ Cited By } & \multirow{2}{*}{$\begin{array}{l}\text { Study } \\
\text { Design }\end{array}$} & \multirow{2}{*}{$\begin{array}{l}\text { Patients } \\
\text { (Implants) }\end{array}$} & \multirow{2}{*}{ Test } & \multirow{2}{*}{ Control } & \multirow{2}{*}{$\begin{array}{l}\text { Marginal Bone Loss } \\
\quad(\text { Mean } \pm \text { SD) }\end{array}$} & \multirow{2}{*}{ Survival Rate } & \multirow{2}{*}{ Failure } & \multirow{2}{*}{$\begin{array}{c}\text { Follow } \\
\text { Up }\end{array}$} \\
\hline & Full Title & Rank & IF & & & & & & & & & \\
\hline $\begin{array}{l}\text { Pirker et al. } \\
(2008)[68]\end{array}$ & $\begin{array}{c}\text { International Journal of } \\
\text { Oral and Maxillofacial } \\
\text { Surgery }\end{array}$ & 33 & 2.068 & 50 & Case report & 1 (1 Implant) & $\begin{array}{l}\text { Microretention and } \\
\text { sandblasted } \\
\text { root-analogue } \\
\text { zirconia implant } \\
\end{array}$ & - & - & $100 \%$ & - & 2 years \\
\hline $\begin{array}{l}\text { Pirker et al. } \\
\text { (2009) [69] }\end{array}$ & $\begin{array}{l}\text { International Journal of } \\
\text { Oral and Maxillofacial } \\
\text { Surgery }\end{array}$ & 33 & 2.068 & 58 & $\begin{array}{l}\text { Prospective } \\
\text { Case Series }\end{array}$ & 18 (18 Implants) & $\begin{array}{l}\text { Microretention and } \\
\text { sandblasted } \\
\text { root-analogue } \\
\text { zirconia implants } \\
\end{array}$ & $\begin{array}{l}\text { Sandblasted } \\
\text { root-analogue } \\
\text { zirconia implants }\end{array}$ & $0.5 \pm 0.7 \mathrm{~mm}$ & $\begin{array}{l}\text { Test: } 92 \% \\
\text { Control: } 0 \%\end{array}$ & $\begin{array}{c}\text { Test: } \\
1 \text { implant } \\
\text { Control: All implants (6) }\end{array}$ & 2 years \\
\hline $\begin{array}{l}\text { Cannizzaro et al. } \\
\quad(2010)[54]\end{array}$ & $\begin{array}{l}\text { European Journal of } \\
\text { Implantology }\end{array}$ & - & - & 69 & $\begin{array}{l}\text { Multicenter } \\
\text { RCT }\end{array}$ & 40 (40 Implants) & $\begin{array}{l}\text { Immediate occlusal } \\
\text { loading zirconia } \\
\text { Implants }\end{array}$ & $\begin{array}{c}\text { Immediate } \\
\text { non-occlusal loading } \\
\text { zirconia Implants }\end{array}$ & $\begin{array}{c}\text { Test: } \\
0.90 \pm 0.48 \mathrm{~mm} \\
\text { Control: } \\
0.72 \pm 0.59 \mathrm{~mm} \\
\end{array}$ & $88.50 \%$ & $\begin{array}{l}5 \text { implants (12.5\%): Test: } \\
3 \text { Implants Control: } \\
2 \text { Implants }\end{array}$ & 1 year \\
\hline $\begin{array}{l}\text { Borgonovo et al. } \\
\quad(2011) \text { [51] }\end{array}$ & Minerva Stomatologica & - & - & 21 & $\begin{array}{l}\text { Prospective } \\
\text { Case Series }\end{array}$ & 16 (26 Implants) & $\begin{array}{c}\text { One-piece yttrium } \\
\text { stabilized zirconia } \\
\text { implants }\end{array}$ & - & - & $96.16 \%$ & $\begin{array}{c}1 \text { Implant } \\
\text { osseointegration failure }\end{array}$ & 2 years \\
\hline $\begin{array}{l}\text { Payer et al. } \\
\text { (2012) [66] }\end{array}$ & $\begin{array}{l}\text { Clinical Oral Implants } \\
\text { Research }\end{array}$ & 8 & 3.723 & 61 & $\begin{array}{l}\text { Prospective } \\
\text { Case Series }\end{array}$ & 20 (20 Implants) & $\begin{array}{l}\text { One-piece zirconia } \\
\text { implants }\end{array}$ & - & $1.29 \pm 0.73 \mathrm{~mm}$ & $95 \%$ & $\begin{array}{c}1 \text { Implant } \\
\text { osseointegration failure }\end{array}$ & 2 years \\
\hline $\begin{array}{l}\text { Akça et al. } \\
\text { (2013) [47] }\end{array}$ & $\begin{array}{l}\text { International Journal of } \\
\text { Oral and Maxillofacial } \\
\text { Implants }\end{array}$ & 24 & 2.32 & 8 & $\begin{array}{l}\text { Prospective } \\
\text { Case Series }\end{array}$ & 23 (52 Implants) & - & - & $0.32 \pm 0.24 \mathrm{~mm}$ & $100 \%$ & No failure & 2 years \\
\hline $\begin{array}{l}\text { Borgonovo et al. } \\
\quad(2013)[52]\end{array}$ & Minerva Stomatologica & - & - & 10 & $\begin{array}{l}\text { Prospective } \\
\text { Case Series }\end{array}$ & 6 (14 Implants) & $\begin{array}{c}\text { One-piece yttrium } \\
\text { stabilized zirconia } \\
\text { implants } \\
\end{array}$ & - & $0.67 \pm 0.51 \mathrm{~mm}$ & $100 \%$ & No failure & 4 years \\
\hline $\begin{array}{l}\text { Kohal et al. } \\
(2013) \text { [59] }\end{array}$ & $\begin{array}{l}\text { Journal of Clinical } \\
\text { Periodontology }\end{array}$ & 2 & 5.241 & 47 & $\begin{array}{l}\text { Prospective } \\
\text { Case Series }\end{array}$ & 28 (56 Implants) & $\begin{array}{c}\text { One-piece } \\
\text { yttria-stabilized } \\
\text { tetragonal zirconia } \\
\text { implants }\end{array}$ & - & $1.95 \pm 0.65$ & $98.20 \%$ & $\begin{array}{c}1 \text { Implant } \\
\text { osseointegration failure }\end{array}$ & 1 year \\
\hline $\begin{array}{l}\text { Osman et al. } \\
\text { (2013) [63] }\end{array}$ & $\begin{array}{l}\text { International Journal of } \\
\text { Prosthodontics }\end{array}$ & 61 & 1.49 & 6 & Pilot study & 4 (28 Implants) & $\begin{array}{c}\text { One-piece zirconia } \\
\text { implants for ball } \\
\text { abutment }\end{array}$ & - & - & $85.70 \%$ & 4 Implants & 1 year \\
\hline $\begin{array}{l}\text { Osman et al. } \\
(2014)[64]\end{array}$ & $\begin{array}{l}\text { Clinical Oral Implants } \\
\text { Research }\end{array}$ & 8 & 3.723 & 34 & RCT & $\begin{array}{l}19(129 \\
\text { Implants) }\end{array}$ & $\begin{array}{l}\text { One-piece zirconia } \\
\text { implants for } \\
\text { ball-abutment }\end{array}$ & $\begin{array}{l}\text { One-piece titanium } \\
\text { implants for } \\
\text { ball-abutment }\end{array}$ & $\begin{array}{c}\text { Zir: } \\
0.42 \pm 0.40 \\
\text { Tit: } \\
0.18 \pm 0.47\end{array}$ & $\begin{array}{l}\text { Zir: } 90.9 \% \text { Tit: } \\
\quad 95.8 \%\end{array}$ & $\begin{array}{c}\text { Zir: } \\
\text { 21Implants } \\
\text { (3 fractured) } \\
\text { Tit: } \\
\text { 10Implants }\end{array}$ & 1 year \\
\hline $\begin{array}{l}\text { Becker et al. } \\
\text { (2015) [50] }\end{array}$ & $\begin{array}{l}\text { Clinical Oral Implants } \\
\text { Research }\end{array}$ & 8 & 3.723 & 15 & $\begin{array}{l}\text { Prospective } \\
\text { Cohort Study }\end{array}$ & 52 (52 Implants) & $\begin{array}{l}\text { Two-piece zirconia } \\
\text { implants }\end{array}$ & - & - & $95.80 \%$ & 2 Implants & 2 years \\
\hline $\begin{array}{l}\text { Cionca et al. } \\
\text { (2015) [55] }\end{array}$ & $\begin{array}{l}\text { Clinical Oral Implants } \\
\text { Research }\end{array}$ & 8 & 3.723 & 43 & $\begin{array}{l}\text { Prospective } \\
\text { Case Series }\end{array}$ & 32 (49 Implants) & $\begin{array}{l}\text { Two-piece zirconia } \\
\text { implants }\end{array}$ & - & - & $87 \%$ & 6 Implants & 1 year \\
\hline
\end{tabular}


Table 2. Cont

\begin{tabular}{|c|c|c|c|c|c|c|c|c|c|c|c|c|}
\hline \multirow{2}{*}{$\begin{array}{c}\text { Authors } \\
\text { (Year) [Ref] }\end{array}$} & \multicolumn{3}{|c|}{ Journal } & \multirow{2}{*}{ Cited By } & \multirow{2}{*}{$\begin{array}{c}\text { Study } \\
\text { Design }\end{array}$} & \multirow{2}{*}{$\begin{array}{c}\text { Patients } \\
\text { (Implants) }\end{array}$} & \multirow{2}{*}{ Test } & \multirow{2}{*}{ Control } & \multirow{2}{*}{$\begin{array}{l}\text { Marginal Bone Loss } \\
\quad \text { (Mean } \pm \text { SD) }\end{array}$} & \multirow{2}{*}{ Survival Rate } & \multirow{2}{*}{ Failure } & \multirow{2}{*}{$\begin{array}{l}\text { Follow } \\
\text { Up }\end{array}$} \\
\hline & Full Title & Rank & IF & & & & & & & & & \\
\hline $\begin{array}{l}\text { Jung et al. } \\
\text { (2015) [56] }\end{array}$ & $\begin{array}{l}\text { Clinical Oral Implants } \\
\text { Research }\end{array}$ & 8 & 3.723 & 27 & $\begin{array}{l}\text { Prospective } \\
\text { Cohort Study }\end{array}$ & 60 (71 Implants) & $\begin{array}{l}\text { Immediate one-piece } \\
\text { zirconia implants }\end{array}$ & - & $0.78 \pm 0.79 \mathrm{~mm}$ & $98.30 \%$ & $\begin{array}{c}1 \mathrm{implant} \\
\text { osseointegration failure }\end{array}$ & 1 year \\
\hline $\begin{array}{l}\text { Payer et al. } \\
\text { (2015) [67] }\end{array}$ & $\begin{array}{l}\text { Clinical Oral Implants } \\
\text { Research }\end{array}$ & 8 & 3.723 & 41 & RCT & 22 (31 Implants) & $\begin{array}{l}\text { Two-piece zirconia } \\
\text { implants }\end{array}$ & $\begin{array}{l}\text { Two-piece titanium } \\
\text { implants }\end{array}$ & $\begin{array}{c}\text { Zir: } \\
1.48 \pm 1.05 \\
\text { Tit: } \\
1.43 \pm 0.67 \\
\end{array}$ & $\begin{array}{c}\text { Zir: } 93.3 \% \\
\text { Tit: } \\
100 \%\end{array}$ & $\begin{array}{c}\text { Zir: } \\
\text { 1 Implant } \\
\text { Tit: } \\
\text { No failure } \\
\end{array}$ & 2 years \\
\hline $\begin{array}{l}\text { Siddiqi et al. } \\
\text { (2015) [70] }\end{array}$ & $\begin{array}{c}\text { Clinical Implant } \\
\text { Dentistry and Related } \\
\text { Research }\end{array}$ & 9 & 3.396 & 17 & RCT & $\begin{array}{l}22(150 \\
\text { Implants) }\end{array}$ & $\begin{array}{l}\text { One-piece zirconia } \\
\text { implants for } \\
\text { ball-abutment }\end{array}$ & $\begin{array}{l}\text { Titanium implants } \\
\text { for one-piece } \\
\text { ball-abutment }\end{array}$ & $\begin{array}{c}\text { Zir: } \\
2.23 \pm 0.69 \\
\text { Tit: } \\
1.59 \pm 0.33 \\
\end{array}$ & $\begin{array}{c}\text { Zir: } 67.6 \% \\
\text { Tit: } \\
66.7 \%\end{array}$ & $\begin{array}{c}\text { Zir: } \\
\text { 16 Implants } \\
\text { Tit: } \\
7 \text { Implants } \\
\end{array}$ & 1 year \\
\hline $\begin{array}{l}\text { Spies et al. } \\
(2015)[73]\end{array}$ & $\begin{array}{l}\text { Journal of Dental } \\
\text { Research }\end{array}$ & 3 & 4.914 & 22 & $\begin{array}{l}\text { Prospective } \\
\text { Cohort Study }\end{array}$ & 40 (53 Implants) & $\begin{array}{c}\text { One-piece } \\
\text { alumina-toughened } \\
\text { zirconia implant }\end{array}$ & - & $0.79 \pm 0.47 \mathrm{~mm}$ & $94.2 \%$ & $\begin{array}{l}3 \text { Implants } \\
\text { osseointegration failure }\end{array}$ & 3 years \\
\hline $\begin{array}{l}\text { Patankar et al. } \\
\text { (2016) [65] }\end{array}$ & $\begin{array}{l}\text { Journal of Maxillofacial } \\
\text { and Oral Surgery }\end{array}$ & - & - & 3 & Case report & 1 (1 Implant) & $\begin{array}{l}\text { Microretention and } \\
\text { sandblasted } \\
\text { root-analogue } \\
\text { zirconia implant }\end{array}$ & - & - & $100 \%$ & - & 1.5 year \\
\hline $\begin{array}{l}\text { Spies et al. } \\
(2016)[74]\end{array}$ & $\begin{array}{l}\text { Clinical Oral Implants } \\
\text { Research }\end{array}$ & 8 & 3.723 & 13 & $\begin{array}{l}\text { Prospective } \\
\text { Cohort Study }\end{array}$ & 27 (27 Implants) & $\begin{array}{l}\text { Immediate one-piece } \\
\text { alumina-toughened } \\
\text { zirconia implant }\end{array}$ & - & $0.77 \pm 0.31 \mathrm{~mm}$ & $88.90 \%$ & $\begin{array}{l}3 \text { Implants } \\
\text { osseointegration failure }\end{array}$ & 1 year \\
\hline $\begin{array}{l}\text { Kniha et al. } \\
\text { (2017) [58] }\end{array}$ & $\begin{array}{l}\text { International Journal of } \\
\text { Oral and Maxillofacial } \\
\text { Surgery } \\
\end{array}$ & 33 & 2.068 & 9 & $\begin{array}{l}\text { Prospective } \\
\text { Cohort Study }\end{array}$ & $\begin{array}{l}81 \text { (105 } \\
\text { Implants) }\end{array}$ & Zirconia implants & - & $0.66 \pm 0.33 \mathrm{~mm}$ & $100 \%$ & No failure & 3 years \\
\hline $\begin{array}{l}\text { Kniha et al. } \\
\text { (2017) [57] }\end{array}$ & $\begin{array}{l}\text { International Journal of } \\
\text { Oral and Maxillofacial } \\
\text { Surgery }\end{array}$ & 33 & 2.068 & 9 & $\begin{array}{l}\text { Prospective } \\
\text { Cohort Study }\end{array}$ & 78 (82 Implants) & $\begin{array}{l}\text { Immediate loading } \\
\text { one-piece zirconia } \\
\text { implants }\end{array}$ & $\begin{array}{l}\text { Delayed one-piece } \\
\text { zirconia implants }\end{array}$ & $\begin{array}{c}\text { Immediate: } \\
0.76 \pm 1.13 \mathrm{~mm} \\
\text { Delayed: } \\
0.83 \pm 0.65 \mathrm{~mm}\end{array}$ & $\begin{array}{c}\text { Immediate: } \\
100 \% \\
\text { Delayed: } \\
100 \%\end{array}$ & No failure & 1 year \\
\hline $\begin{array}{l}\text { Spies et al. } \\
\text { (2017) [71] }\end{array}$ & Journal of Dentistry & 10 & 3.242 & 6 & $\begin{array}{l}\text { Prospective } \\
\text { Case Series }\end{array}$ & 60 (71 Implants) & $\begin{array}{l}\text { One-piece zirconia } \\
\text { oral implants }\end{array}$ & - & - & $100 \%$ & No failure & 3 years \\
\hline $\begin{array}{l}\text { Spies et al. } \\
(2017)[75]\end{array}$ & $\begin{array}{l}\text { Clinical Oral Implants } \\
\text { Research }\end{array}$ & 8 & 3.723 & 11 & $\begin{array}{l}\text { Prospective } \\
\text { Case Series }\end{array}$ & 13 (26 Implants) & $\begin{array}{l}\text { One-piece zirconia } \\
\text { implants }\end{array}$ & - & - & $100 \%$ & No failure & 5 years \\
\hline $\begin{array}{l}\text { Balmer et al. } \\
(2018)[49]\end{array}$ & $\begin{array}{l}\text { Clinical Oral Implants } \\
\text { Research }\end{array}$ & 8 & 3.723 & 11 & $\begin{array}{l}\text { Prospective } \\
\text { Multicenter } \\
\text { Cohort Study }\end{array}$ & 60 (71 Implants) & $\begin{array}{l}\text { One-piece immediate } \\
\text { loading zirconia } \\
\text { implants }\end{array}$ & - & $0.70 \pm 0.72 \mathrm{~mm}$ & $98.50 \%$ & $\begin{array}{c}1 \text { Implant } \\
\text { osseointegration failure }\end{array}$ & 3 years \\
\hline $\begin{array}{l}\text { Bormann et al. } \\
\text { (2018) [53] }\end{array}$ & BMC Oral Health & 38 & 1.911 & 7 & $\begin{array}{l}\text { Prospective } \\
\text { Multicenter } \\
\text { Cohort Study }\end{array}$ & 44 (44 Implants) & Zirconia implants & - & $0.97 \pm 0.88 \mathrm{~mm}$ & $97.50 \%$ & 1 Implant & 3 years \\
\hline $\begin{array}{l}\text { Kohal et al. } \\
\text { (2018) [60] }\end{array}$ & $\begin{array}{l}\text { Journal of Clinical } \\
\text { Periodontology }\end{array}$ & 2 & 5.241 & 5 & $\begin{array}{l}\text { Prospective } \\
\text { Cohort Study }\end{array}$ & 65 (65 Implants) & $\begin{array}{l}\text { Immediate loading } \\
\text { one-piece zirconia } \\
\text { implants }\end{array}$ & - & $1.45 \pm 1.96 \mathrm{~mm}$ & $90.80 \%$ & 6 Implants & 3 years \\
\hline
\end{tabular}


Table 2. Cont.

\begin{tabular}{|c|c|c|c|c|c|c|c|c|c|c|c|c|}
\hline \multirow{2}{*}{$\begin{array}{l}\text { Authors } \\
\text { (Year) [Ref] }\end{array}$} & \multicolumn{3}{|c|}{ Journal } & \multirow{2}{*}{ Cited By } & \multirow{2}{*}{$\begin{array}{l}\text { Study } \\
\text { Design }\end{array}$} & \multirow{2}{*}{$\begin{array}{l}\text { Patients } \\
\text { (Implants) }\end{array}$} & \multirow{2}{*}{ Test } & \multirow{2}{*}{ Control } & \multirow{2}{*}{$\begin{array}{l}\text { Marginal Bone Loss } \\
\quad \text { (Mean } \pm \text { SD) }\end{array}$} & \multirow{2}{*}{ Survival Rate } & \multirow{2}{*}{ Failure } & \multirow{2}{*}{$\begin{array}{l}\text { Follow } \\
\text { Up }\end{array}$} \\
\hline & Full Title & Rank & IF & & & & & & & & & \\
\hline $\begin{array}{l}\text { Lorenz et al. } \\
\text { (2019) [62] }\end{array}$ & $\begin{array}{c}\text { Clinical Implant } \\
\text { Dentistry and Related } \\
\text { Research } \\
\end{array}$ & 9 & 3.396 & 4 & $\begin{array}{l}\text { Prospective } \\
\text { Cohort Study }\end{array}$ & 28 (83 Implants) & Zirconia implants & Natural teeth & $1.2 \pm 0.76 \mathrm{~mm}$ & $100 \%$ & $\begin{array}{c}\text { No failure one } \\
\text { peri-implantitis resistant } \\
\text { to therapies }\end{array}$ & 7.8 years \\
\hline $\begin{array}{l}\text { Spies et al. } \\
\text { (2019) [72] }\end{array}$ & $\begin{array}{l}\text { Clinical Oral Implants } \\
\text { Research }\end{array}$ & 8 & 3.723 & 5 & $\begin{array}{l}\text { Prospective } \\
\text { Multicenter } \\
\text { Cohort Study }\end{array}$ & 45 (45 Implants) & Zirconia implants & - & - & $97.5 \pm 2.47 \%$ & $\begin{array}{l}\text { Chipping }(\mathrm{n}=19) \\
\text { occlusal roughness } \\
(\mathrm{n}=35)\end{array}$ & 5 years \\
\hline $\begin{array}{l}\text { Balmer et al. } \\
(2020)[48]\end{array}$ & $\begin{array}{l}\text { Clinical Oral Implants } \\
\text { Research }\end{array}$ & 8 & 3.723 & 4 & $\begin{array}{l}\text { Prospective } \\
\text { Multicenter } \\
\text { Cohort Study }\end{array}$ & 60 (71 Implants) & $\begin{array}{l}\text { Single crown } \\
\text { one-piece zirconia } \\
\text { implant }\end{array}$ & $\begin{array}{l}\text { Multiple prostheses } \\
\text { one-piece zirconia } \\
\text { implant }\end{array}$ & $0.7 \pm 0.6 \mathrm{~mm}$ & $98.4 \%$ & 1 Implant & 5 years \\
\hline $\begin{array}{l}\text { Koller et al. } \\
\text { (2020) [61] }\end{array}$ & $\begin{array}{l}\text { Clinical Oral Implants } \\
\text { Research }\end{array}$ & 8 & 3.723 & 0 & Pilot RCT & 22 (31 Implants) & $\begin{array}{l}\text { Two-piece zirconia } \\
\text { implants }\end{array}$ & $\begin{array}{l}\text { Two-piece titanium } \\
\text { implants }\end{array}$ & $\begin{array}{c}\text { Zir: } \\
1.38 \pm 0.81 \mathrm{Tit:} \\
1.17 \pm 0.73 \mathrm{~mm}\end{array}$ & $\begin{array}{l}\text { Zir: } 87.5 \% \text { Tit: } \\
\quad 93.3 \%\end{array}$ & $\begin{array}{l}\text { Zir: } \\
2 \text { Implants } \\
\text { Tit: } \\
1 \text { Implant }\end{array}$ & 6.67 years \\
\hline
\end{tabular}




\subsection{Growth of Publications}

In total, 29 clinical studies were published between 2008 and 2020, in which 19 papers (65.5\%) were published in the last five years and ten papers published before 2015. The highest number of published studies was in 2015 ( $n=6,20.6 \%)$ followed by 2013 and 2017 ( $n=4,13.7 \%$ for each) (Figure 2).

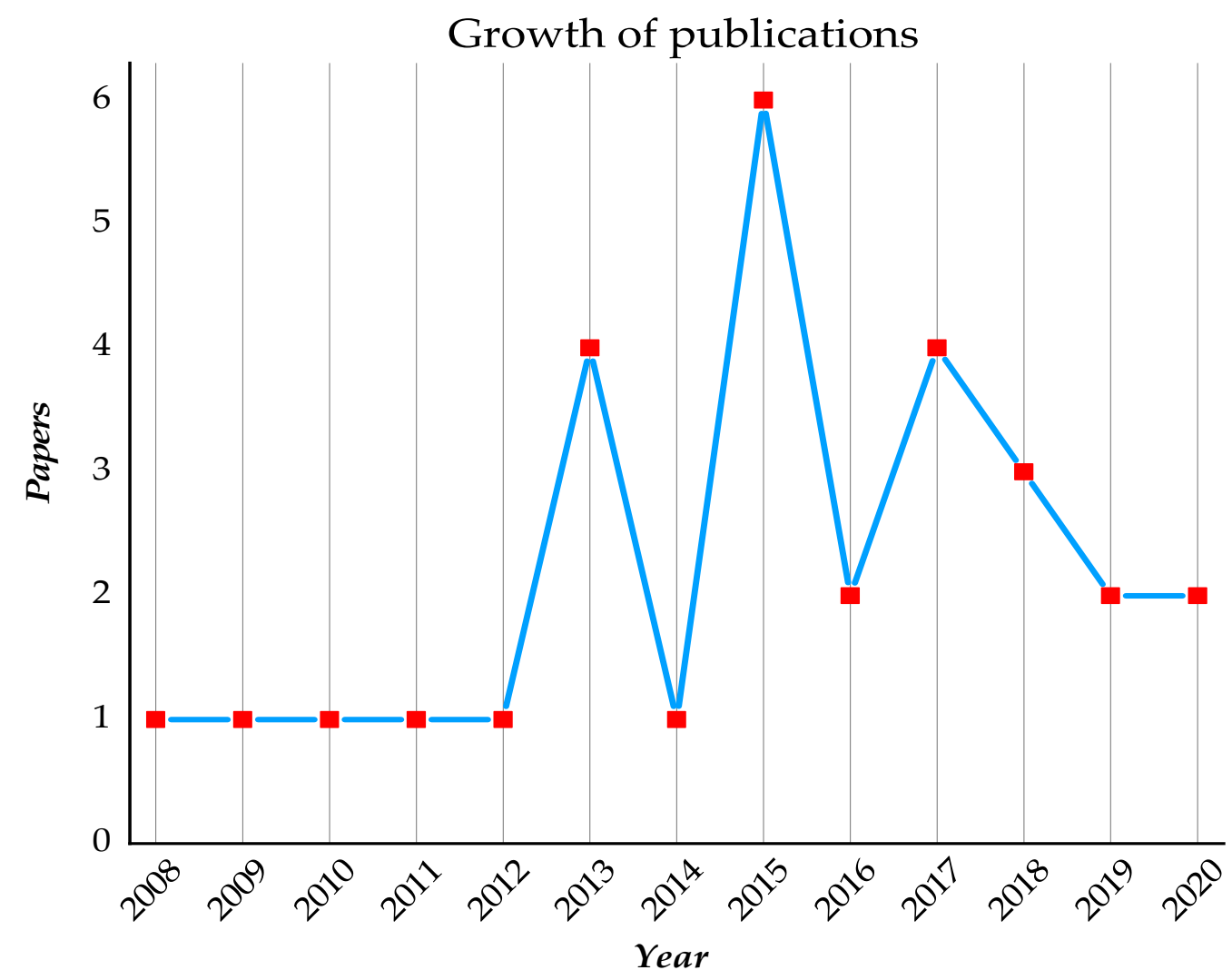

Figure 2. Publication trend of the clinical studies on the zirconia implants.

\subsection{Journal of Publication}

The clinical studies on the use of zirconia dental implants for oral rehabilitation were published across ten peer-reviewed journals. The journal with the largest number of publications was "Clinical Oral Implants Research" ( $n=12,41 \%)$, followed by "International Journal of Oral and Maxillofacial Surgery" $(n=4,13.7 \%)$ (Figure 3$)$.

The majority of publications were published in Q1 journals $(n=25,86 \%)$, while the journal with the highest impact factor was "Journal of Clinical Periodontology" (IF = 5.241), which had two articles. 


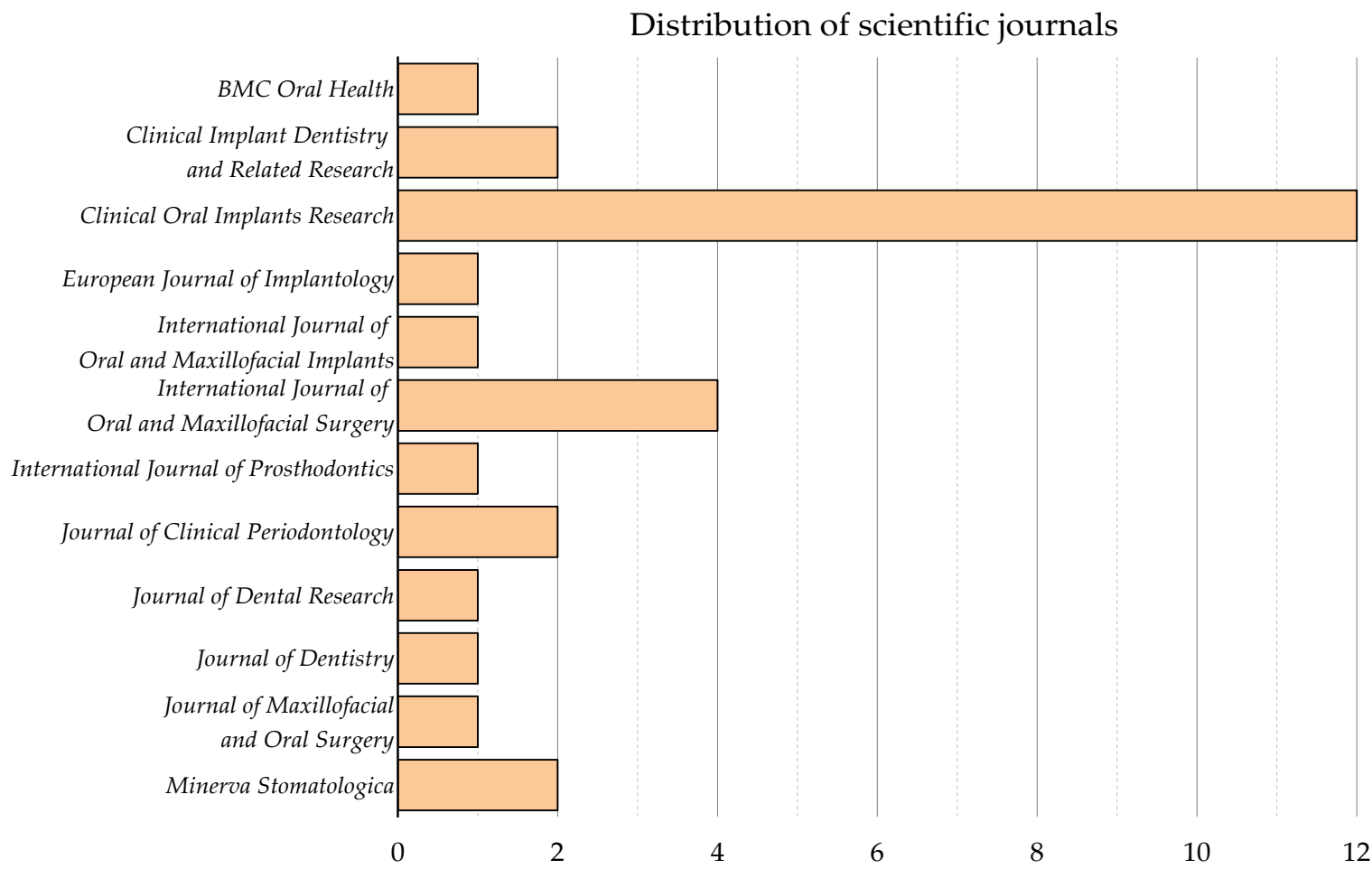

Figure 3. Contribution journals in clinical research on zirconia implants.

\subsection{Study Design and Level of Evidence}

All included studies were prospective, while the most common study design of clinical research on zirconia implants was cohort study $(n=12,41.4 \%)$, followed by case series $(n=9,31 \%)$, and RCT $(n=5$, $17 \%)$. According to the hierarchy of evidence levels (Is) [77,78], the available evidence supporting the use of zirconia implants is $17 \%$ level II, $41.4 \%$ EL IV, and the remaining EL VI.

\subsection{Contribution of Countries and Institutions}

The majority of the studies originated from institutions in Germany ( $n=14,48.3 \%)$, followed by Switzerland, $(n=6,20.7 \%)$, and Austria $(n=5,17 \%)$, where the most productive institution was the Medical Center of University of Freiburg $(n=8,27.6 \%)$, followed by the Center of Dental Medicine, University of Zürich ( $n=5,17 \%)$. While many of the included studies were funded, the most funding support for included research was provided by VITA Zahnfabrik- $\mathrm{H}$. Rauter GmbH \& Co. KG, Bad Säckingen, Germany ( $n=5,17 \%$ ) (Table 3). 
Table 3. Contribution of countries and institutions to clinical studies on zirconia implants.

\begin{tabular}{|c|c|c|c|}
\hline Country & Institution & Study [Ref] & Funding \\
\hline \multirow{10}{*}{ Germany } & Universitätsklinikum Düsseldorf, Düsseldorf & Becker et al., 2015 [50] & ZV3 Zircon Vision GmbH, Wolfratshausen, Germany \\
\hline & University Hospital Aachen, Aachen & Kniha et al., 2017 [57] & \multirow{3}{*}{ No Funding } \\
\hline & Friedrich-Alexander-University Erlangen-Nürnberg & Kniha et al., 2017 [58] & \\
\hline & Johann-Wolfgang Goethe University, Frankfurt/Main & Lorenz et al., 2019 [62] & \\
\hline & Hannover Medical School, Hannover & Bormann et al., 2018 [53] & Institut Straumann AG, Basel, Switzerland \\
\hline & School of Dentistry, Albert-Ludwigs University, Freiburg & Kohal et al., 2013 [59] & \multirow{2}{*}{ Nobel Biocare AB, Göteborg, Sweden } \\
\hline & \multirow{4}{*}{ Medical Center of University of Freiburg, Freiburg } & Kohal et al., 2018 [60] & \\
\hline & & Spies et al., 2015 [73] & Metoxit AG (Thayngen, Switzerland) \\
\hline & & Spies et al., 2016 [74] & \\
\hline & & Spies et al., 2017 [75] & Ivoclar Vivadent \\
\hline \multirow{4}{*}{ Germany and Switzerland } & \multirow{4}{*}{$\begin{array}{l}\text { Medical Center of University of Freiburg, Freiburg and } \\
\text { Center of Dental Medicine, University of Zürich, Zürich }\end{array}$} & Spies et al., 2017 [71] & \multirow{4}{*}{ VITA Zahnfabrik-H. Rauter GmbH \& Co. KG, Bad Säckingen, Germany } \\
\hline & & Balmer et al., 2018 [49] & \\
\hline & & Spies et al., 2019 [72] & \\
\hline & & Balmer et al., 2020 [48] & \\
\hline \multirow{2}{*}{ Switzerland } & School of Dental Medicine, University of Geneva, Geneva & Cionca et al., 2015 [55] & Dentalpoint AG, Zürich, Switzerland \\
\hline & Center of Dental Medicine, University of Zürich, Zürich & Jung et al., 2015 [56] & VITA Zahnfabrik-H. Rauter GmbH \& Co. KG, Bad Säckingen, Germany \\
\hline \multirow{5}{*}{ Austria } & \multirow{2}{*}{ Alfred Kocher, Medical University Vienna, Vienna } & Pirker et al., 2008 [68] & \multirow{2}{*}{ No Funding } \\
\hline & & Pirker et al., 2009 [69] & \\
\hline & \multirow{3}{*}{ School of Dentistry, Medical University Graz, Graz } & Payer et al., 2012 [66] & Bredent medical GmbH, Senden, Germany \\
\hline & & Payer et al., 2015 [67] & \multirow{2}{*}{ Ziterion $\mathrm{GmbH}$, Uffenheim, Germany } \\
\hline & & Koller et al., 2020 [61] & \\
\hline \multirow{3}{*}{ Italy } & Private practice & Cannizzaro et al., 2010 [54] & Partially supported by Z-systems \\
\hline & \multirow{2}{*}{ School of Dentistry, University of Milan, Milan } & Borgonovo et al., 2011 [51] & \multirow{2}{*}{ Not reported } \\
\hline & & Borgonovo et al., 2013 [52] & \\
\hline \multirow{3}{*}{ New Zealand } & \multirow{3}{*}{$\begin{array}{l}\text { Oral Implantology Research Group, Sir John Walsh } \\
\text { Research Institute, School of Dentistry, University of Otago }\end{array}$} & Osman et al., 2013 [63] & \multirow{3}{*}{$\begin{array}{l}\text { Oral Implantology Research Group, Sir John Walsh Research Institute, } \\
\text { School of Dentistry, University of Otago and Southern Implants }\end{array}$} \\
\hline & & Osman et al., 2014 [64] & \\
\hline & & Siddiqi et al., 2015 [70] & \\
\hline India & BV Dental College and Hospital, Pune & Patankar et al., 2016 [65] & No Funding \\
\hline Turkey & Faculty of Dentistry, Hacettepe University & Akça et al., 2013 [47] & No Funding \\
\hline
\end{tabular}




\subsection{Bibliometric Assessment}

A total of 29 articles with total citations[scopus] ranged from 0 to 176 (mean $57.28 \pm 42.18$ ), while the number of citations [Scopus] received by each paper ranged from 0 to 69 (mean $21.3 \pm 20$ ). The top-cited study was the RCT of Cannizzaro et al. (2010) ( $\left.n_{\text {[Scopus] }}=69\right)$ [54], followed by the prospective case series of Payer et al. (2012) $\left(n_{\text {[Scopus] }}=61\right)$ [66], and Pirker et al. $(2009)\left(n_{[\text {Scopus] }}=58\right)$ [69].

However, the author with the highest number of clinical research on zirconia implants was Kohal R.J. $(n=10)$, followed by Spies B.C. $(n=8)$ and Vach K. $(n=6)$, while the top-cited author of clinical studies on zirconia implants was Kohal R.J. $\left(n_{[\mathrm{WoS}]}=155\right)$, followed by Arnetzl G. $\left(n_{[\mathrm{WoS}]}=91\right)$, Koller M. $\left(n_{[\mathrm{WoS}]}=87\right)$, Payer M., and Jakse N. $\left(n_{[\mathrm{WoS}]}=86\right.$ for each) (Figures 4 and 5$)$.

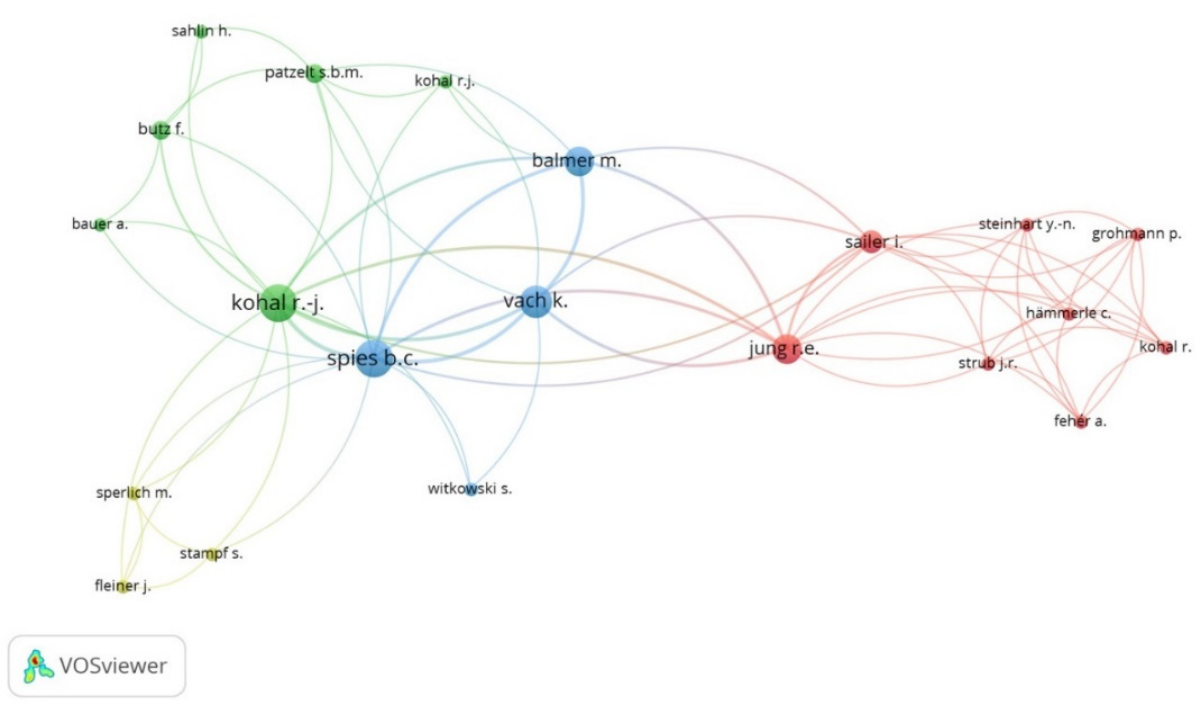

Figure 4. Network analysis of the authors with the largest number of clinical studies on zirconia implants.

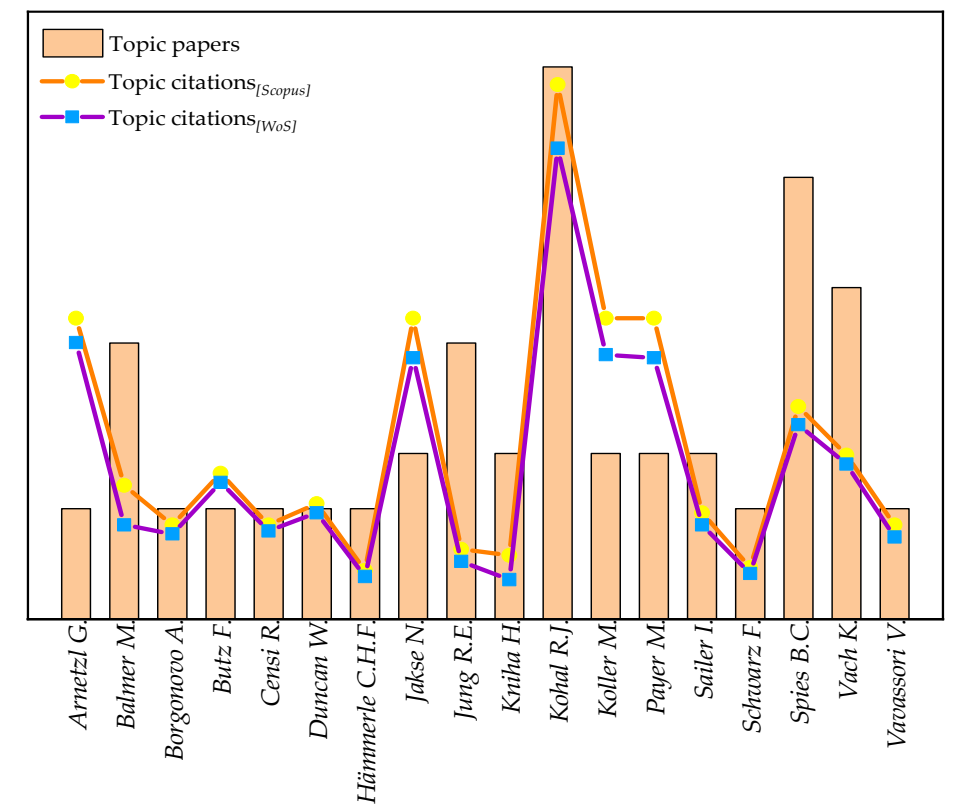

Figure 5. Bibliometric variables for authors with the highest number of topic papers.

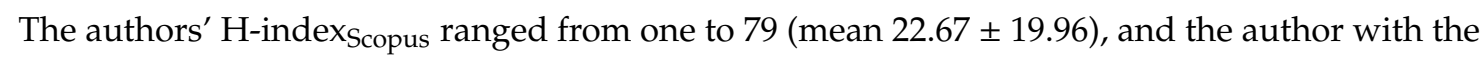
most bibliometric characteristics was Hämmerle C.H.F., who had 364 publications (two of which were clinical studies on zirconia implants) with 8311 total citations and H-index Scopus $=79$ (Table 4 and Figure 6). 
Table 4. General bibliometric variables for authors with the largest number of topic papers.

\begin{tabular}{|c|c|c|c|c|c|c|c|c|c|c|}
\hline Author & $\begin{array}{l}\text { Topic } \\
\text { Papers }\end{array}$ & Total Papers & $\begin{array}{c}\text { Topic/Total } \\
\text { Papers \% }\end{array}$ & $\begin{array}{c}\text { Topic } \\
\text { Citations } \\
\text { [Scopus] }\end{array}$ & $\begin{array}{c}\text { Topic } \\
\text { Citations } \\
\text { [WoS] }\end{array}$ & $\begin{array}{c}\text { Total } \\
\text { Citations } \\
\text { [Scopus] }\end{array}$ & $\begin{array}{c}\text { Total Citation } \\
\text { [WoS] }\end{array}$ & $\begin{array}{c}\text { Topic/Total } \\
\text { Citations \% } \\
\text { [Scopus-WoS] }\end{array}$ & $\begin{array}{l}\text { H-index } \\
\text { [Scopus] }\end{array}$ & $\begin{array}{c}\text { H-index } \\
\text { [WoS] }\end{array}$ \\
\hline Kohal R.J. & 10 & 109 & $9.17 \%$ & 176 & 155 & 3053 & 2975 & {$[5.76-5.21 \%]$} & 30 & 29 \\
\hline Spies B.C. & 8 & 47 & $17.02 \%$ & 70 & 64 & 452 & 448 & [15.49-14.29\%] & 14 & 14 \\
\hline Vach K. & 6 & 73 & $8.22 \%$ & 54 & 51 & 661 & 820 & {$[8.17-6.21 \%]$} & 15 & 16 \\
\hline Balmer M. & 5 & 13 & $38.46 \%$ & 44 & 31 & 256 & 130 & [17.19-23.84\%] & 7 & 5 \\
\hline Jung R.E. & 5 & 202 & $2.48 \%$ & 23 & 19 & 8359 & 9126 & {$[0.28-0.21 \%]$} & 47 & 57 \\
\hline Jakse N. & 3 & 70 & $4.29 \%$ & 99 & 86 & 1114 & 981 & [8.89-8.77\%] & 18 & 18 \\
\hline Kniha H. & 3 & 28 & $10.71 \%$ & 21 & 13 & 664 & 726 & {$[3.16-1.779 \%]$} & 11 & 13 \\
\hline Koller M. & 3 & 9 & $33.33 \%$ & 99 & 87 & 166 & 312 & [59.64-27.88\%] & 5 & 5 \\
\hline Payer M. & 3 & 48 & $6.25 \%$ & 99 & 86 & 770 & 1484 & {$[12.86-5.79 \%]$} & 15 & 23 \\
\hline Sailer I. & 3 & 113 & $2.65 \%$ & 35 & 31 & 6027 & 5713 & {$[0.58-0.54 \%]$} & 34 & 33 \\
\hline Arnetzl G. & 2 & 40 & $5.00 \%$ & 99 & 91 & 437 & 183 & {$[22.65-49.72 \%]$} & 11 & 6 \\
\hline Butz F. & 2 & 24 & $8.33 \%$ & 48 & 45 & 1248 & 1441 & {$[3.85-3.12 \%]$} & 18 & 19 \\
\hline Censi R. & 2 & 18 & $11.11 \%$ & 31 & 29 & 169 & 95 & [18.34-30.52\%] & 8 & 4 \\
\hline Duncan W. & 2 & 87 & $2.30 \%$ & 38 & 35 & 2163 & 1356 & {$[1.76-2.58 \%]$} & 20 & 19 \\
\hline Hämmerle C.H.F. & 2 & 364 & $0.55 \%$ & 16 & 14 & 18,311 & 16,032 & [0.09-0.08\%] & 79 & 72 \\
\hline Schwarz F. & 2 & 261 & $0.77 \%$ & 17 & 15 & 9093 & 9169 & [0.19-0.16\%] & 57 & 57 \\
\hline Vavassori V. & 2 & 12 & $16.67 \%$ & 31 & 27 & 136 & 96 & {$[22.79-28.12 \%]$} & 7 & 5 \\
\hline Borgonovo A. & 2 & 51 & $3.92 \%$ & 31 & 28 & 535 & 449 & {$[5.79-6.23 \%]$} & 12 & 11 \\
\hline $\begin{array}{c}\text { Summary } \\
(\text { Mean } \pm \text { SD) }\end{array}$ & $3.61 \pm 2.33$ & $87.17 \pm 96.37$ & $10.07 \pm 0.11 \%$ & $57.28 \pm 42.18$ & $50.39 \pm 37.68$ & $2978.56 \pm 4750.05$ & $2863.11 \pm 4386.90$ & - & $22.67 \pm 19.96$ & $22.56 \pm 20.14$ \\
\hline
\end{tabular}




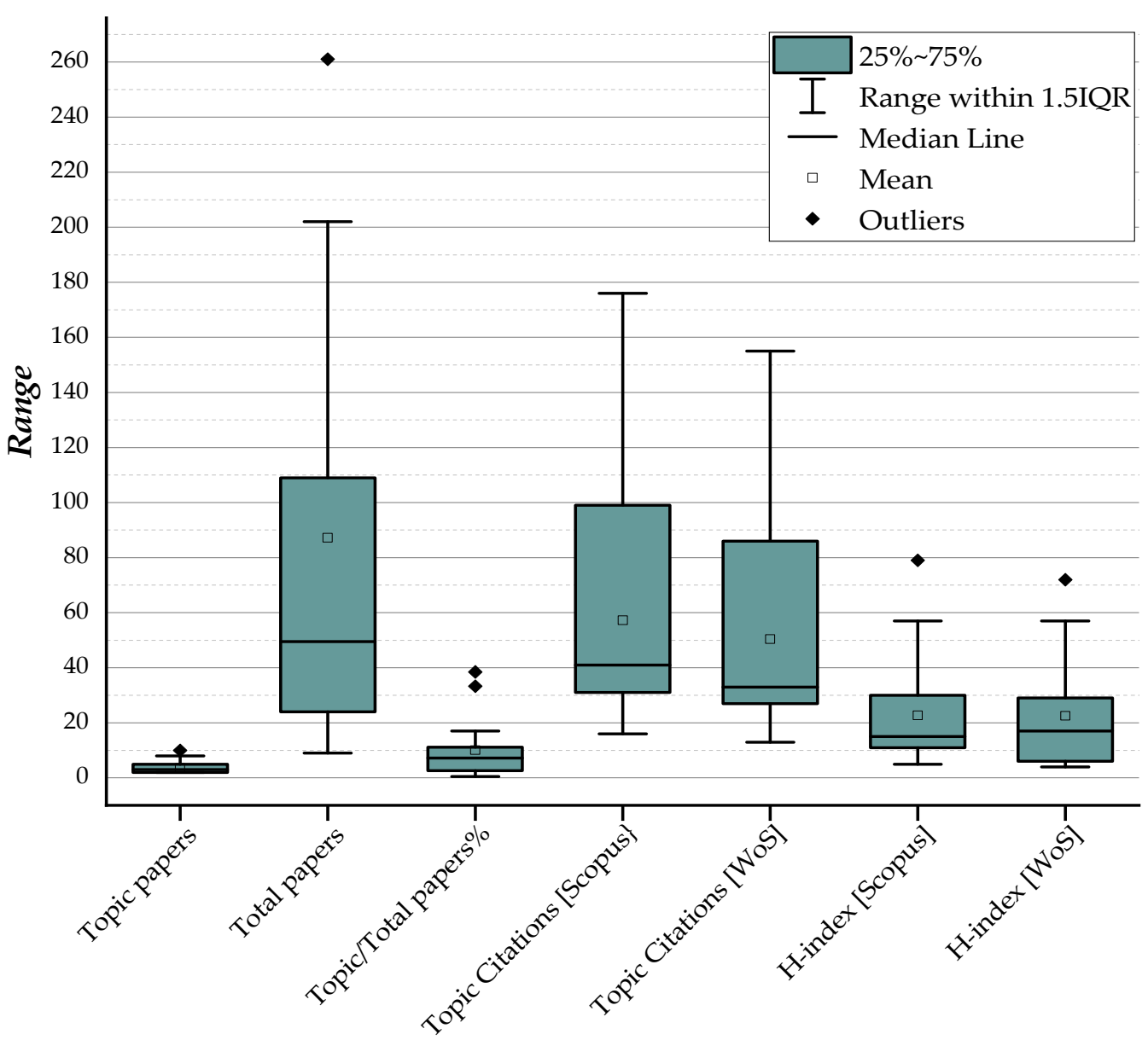

Figure 6. Box plots summarize the bibliometric variables of the authors with the largest number of studies.

\section{Discussion}

The present study carried out a bibliometric evaluation of clinical research on zirconia implant rehabilitation, highlighting the significant heterogeneity of the included studies, which revealed considerable variations in methodology, technical approaches, follow-up, and control group involvement. Our findings indicate that there is a trend for zirconia implants in oral rehabilitation as there has been an increase in about $180 \%$ of the studies published in the last five years.

The included studies reported a survival rate for zirconia implants ranging from $87 \%$ to $100 \%$ with follow-up periods from one to 7.8 years, while the least survival rate reported in RCT by Siddiqi et al. was $67.6 \%$ after one-year follow-up (i.e., 16 zirconia implants failed out of 68) [70]. This RCT aimed to study the effectiveness of zirconia vs. titanium implants restored with one-piece ball-abutment in mandibular and maxillary overdentures, while this high decrease in the survival rate was for both groups (i.e., $67.6 \%$ for zirconia implants and $66.7 \%$ for titanium implants); the outcomes of maxillary rehabilitation were worse than the mandible, while no mechanical fractures of the fixtures were reported [70].

Although one-piece and two-piece zirconia implants have been evaluated, the lower marginal bone loss and higher survival rates were observed in studies of one-piece zirconia implant rehabilitation on a single tooth or three element prosthetic rehabilitation $[59,61]$. However, the studies did not report any differences in the marginal bone loss and survival rate between the single crown and the fixed multiple zirconia implant recovery, while the prosthetic connection appears to have no apparent effect on these parameters [48]. Additionally, Lorenz et al. showed no significant difference in marginal bone loss with a total of 83 zirconia implants compared to natural teeth after 7.8 years of function [47], and the marginal bone loss was similar in the other studies, which was less than $1 \mathrm{~mm}$ in the first year and 
stabilized in subsequent functional loading [47-49,52,56-58,64,69,73,74]. Moreover, the prospective study by Kniha et al. contained the largest sample size of the included studies involving 81 patients with 105 implants for fixed rehabilitation, who reported a significant decrease of $0.66 \pm 0.30 \mathrm{~mm}$ with a survival rate of $100 \%$ after three years [58].

However, the most common complication $(70 \%)$ was the failure of implant osseointegration as 17 studies reported a loss of at least one implant in the first six months [48-51,53-56,59-61,63,64,66,67, $69,70,72-74]$.

As previously reported for titanium dental implant threads, microgeometry appears to have a significant effect on the osseointegration of zirconia implants $[79,80]$, whereas a more retentive surface resulted in an increased survival rate compared to a sandblasted surface only $[68,69]$.

Although all clinical research included in this analysis was screened and selected from the Scopus and Web of Science databases, which may avoid restriction in each database $[39,81]$, our investigation has further limitations. First, the year of publication, which is a reliable indicator of the number of citations received, as older papers receive more citations than recent publications because there is more time to cite them, regardless of their impact [82,83]. Second, open access policies have a significant influence on the citations received in the evaluated papers [84-86], as a result, we found large heterogeneity in Topic/Total Citations\% and co-authors' H-index.

\section{Conclusions}

This was the first study highlighting bibliometric output of clinical research and co-authors in the field of zirconia dental implants and shows a strong interest in the development of research into the clinical application of zirconia dental implants, as evidenced by the increase in the number of scientific papers published in the last ten years.

Author Contributions: Conceptualization, F.L. and A.S.; Methodology, F.L. and A.G.A.K.; Software, F.L.; Validation, F.L. and A.S.; Formal analysis, F.L. and A.G.A.K.; Investigation, A.S., F.L. and B.R.; Data curation, F.L., A.G.A.K. and A.S.; Writing-original draft preparation, F.L. and A.S.; Writing-review and editing, F.L., A.G.A.K. and S.N.; Visualization, A.S., B.R. and I.F.; Supervision, A.S. and I.F. All authors have read and agreed to the published version of the manuscript.

Funding: This research received no external funding.

Conflicts of Interest: Authors declare no conflicts of interest.

\section{References}

1. Albrektsson, T.; Wennerberg, A. On osseointegration in relation to implant surfaces. Clin. Implant Dent. Relat. Res. 2019, 21 (Suppl. 1), 4-7. [CrossRef]

2. Buser, D.; Janner, S.F.M.; Wittneben, J.-G.; Brägger, U.; Ramseier, C.A.; Salvi, G.E. 10-Year Survival and Success Rates of 511 Titanium Implants with a Sandblasted and Acid-Etched Surface: A Retrospective Study in 303 Partially Edentulous Patients. Clin. Implant Dent. Relat. Res. 2012, 14, 839-851. [CrossRef] [PubMed]

3. Degidi, M.; Piattelli, A. A 7-year Follow-up of 93 Immediately Loaded Titanium Dental Implants. J. Oral Implantol. 2005, 31, 25-31. [CrossRef] [PubMed]

4. Scarano, A.; Inchingolo, F.; Murmura, G.; Traini, T.; Piattelli, A.; Lorusso, F. Three-Dimensional Architecture and Mechanical Properties of Bovine Bone Mixed with Autologous Platelet Liquid, Blood, or Physiological Water: An In Vitro Study. Int. J. Mol. Sci. 2018, 19. [CrossRef] [PubMed]

5. Barros, R.R.M.; Degidi, M.; Novaes, A.B.; Piattelli, A.; Shibli, J.A.; Iezzi, G. Osteocyte Density in the Peri-Implant Bone of Immediately Loaded and Submerged Dental Implants. J. Periodontol. 2009, 80, 499-504. [CrossRef]

6. Gehrke, S.; Mazón, P.; Del Fabbro, M.; Tumedei, M.; Aramburú Júnior, J.; Pérez-Díaz, L.; De Aza, P. Histological and Histomorphometric Analyses of Two Bovine Bone Blocks Implanted in Rabbit Calvaria. Symmetry 2019, 11, 641. [CrossRef] 
7. Scarano, A.; Carinci, F.; Lorusso, F.; Festa, F.; Bevilacqua, L.; Santos de Oliveira, P.; Maglione, M. Ultrasonic vs Drill Implant Site Preparation: Post-Operative Pain Measurement Through VAS, Swelling and Crestal Bone Remodeling: A Randomized Clinical Study. Mater. Basel 2018, 11, 2516. [CrossRef]

8. Scarano, A.; Crincoli, V.; Di Benedetto, A.; Cozzolino, V.; Lorusso, F.; Podaliri Vulpiani, M.; Grano, M.; Kalemaj, Z.; Mori, G.; Grassi, F.R. Bone Regeneration Induced by Bone Porcine Block with Bone Marrow Stromal Stem Cells in a Minipig Model of Mandibular “Critical Size" Defect. Stem. Cells Int. 2017, 2017, 9082869. [CrossRef]

9. Scarano, A.; De Oliveira, P.S.; Traini, T.; Lorusso, F. Sinus Membrane Elevation with Heterologous Cortical Lamina: A Randomized Study of a New Surgical Technique for Maxillary Sinus Floor Augmentation without Bone Graft. Mater. Basel 2018, 11, 1457. [CrossRef]

10. Piattelli, A.; Scarano, A.; Piattelli, M. Detection of alkaline and acid phosphatases around titanium implants: A light microscopical and histochemical study in rabbits. Biomaterials. 1995, 16, 1333-1338. [CrossRef]

11. Comuzzi, L.; Tumedei, M.; Piattelli, A.; Iezzi, G. Short vs. Standard Length Cone Morse Connection Implants: An In Vitro Pilot Study in Low Density Polyurethane Foam. Symmetry 2019, 11, 1349. [CrossRef]

12. Tumedei, M.; Savadori, P.; Del Fabbro, M. Synthetic Blocks for Bone Regeneration: A Systematic Review and Meta-Analysis. Int. J. Mol. Sci. 2019, 20, 4221. [CrossRef] [PubMed]

13. Afrashtehfar, K.I.; Del Fabbro, M. Clinical performance of zirconia implants: A meta-review. J. Prosthet. Dent. 2020, 123, 419-426. [CrossRef] [PubMed]

14. Hanawa, T. Zirconia versus titanium in dentistry: A review. Dent. Mater. J. 2020, 39, 24-36. [CrossRef] [PubMed]

15. Hashim, D.; Cionca, N.; Courvoisier, D.S.; Mombelli, A. A systematic review of the clinical survival of zirconia implants. Clin. Oral Investig. 2016, 20, 1403-1417. [CrossRef]

16. Cantore, S.; Mirgaldi, R.; Ballini, A.; Coscia, M.F.; Scacco, S.; Papa, F.; Inchingolo, F.; Dipalma, G.; De Vito, D. Cytokine gene polymorphisms associate with microbiogical agents in periodontal disease: Our experience. Int. J. Med. Sci. 2014, 11, 674-679. [CrossRef]

17. Noronha Oliveira, M.; Schunemann, W.V.H.; Mathew, M.T.; Henriques, B.; Magini, R.S.; Teughels, W.; Souza, J.C.M. Can degradation products released from dental implants affect peri-implant tissues? J. Periodontal Res. 2017, 53,1-11. [CrossRef]

18. Noumbissi, S.; Scarano, A.; Gupta, S. A Literature Review Study on Atomic Ions Dissolution of Titanium and Its Alloys in Implant Dentistry. Mater. Basel 2019, 12, 368. [CrossRef]

19. Ottria, L.; Lauritano, D.; Andreasi Bassi, M.; Palmieri, A.; Candotto, V.; Tagliabue, A.; Tettamanti, L. Mechanical, chemical and biological aspects of titanium and titanium alloys in implant dentistry. J. Biol. Regul. Homeost. Agents 2018, 32 (Suppl. 1), 81-90.

20. Rodrigues, D.C.; Valderrama, P.; Wilson, T.G.; Palmer, K.; Thomas, A.; Sridhar, S.; Adapalli, A.; Burbano, M.; Wadhwani, C. Titanium Corrosion Mechanisms in the Oral Environment: A Retrieval Study. Mater. Basel 2013, 6, 5258-5274. [CrossRef]

21. Scarano, A.; Piattelli, M.; Caputi, S.; Favero, G.A.; Piattelli, A. Bacterial Adhesion on Commercially Pure Titanium and Zirconium Oxide Disks: An In Vivo Human Study. J. Periodontol. 2004, 75, 292-296. [CrossRef] [PubMed]

22. Mihatovic, I.; Golubovic, V.; Becker, J.; Schwarz, F. Bone tissue response to experimental zirconia implants. Clin. Oral Investig. 2016, 21, 523-532. [CrossRef] [PubMed]

23. Scarano, A.; Di Carlo, F.; Quaranta, M.; Piattelli, A. Bone Response to Zirconia Ceramic Implants: An Experimental Study in Rabbits. J. Oral Implantol. 2003, 29, 8-12. [CrossRef]

24. Agustín-Panadero, R.; Serra-Pastor, B.; Roig-Vanaclocha, A.; Fons-Font, A.; Solá-Ruiz, M.F. Fracture resistance and the mode of failure produced in metal-free crowns cemented onto zirconia abutments in dental implants. PLoS ONE 2019, 14, e0220551. [CrossRef] [PubMed]

25. Bethke, A.; Pieralli, S.; Kohal, R.-J.; Burkhardt, F.; von Stein-Lausnitz, M.; Vach, K.; Spies, B.C. Fracture Resistance of Zirconia Oral Implants In Vitro: A Systematic Review and Meta-Analysis. Mater. Basel 2020, 13, 562. [CrossRef] [PubMed]

26. Sailer, I.; Asgeirsson, A.G.; Thoma, D.S.; Fehmer, V.; Aspelund, T.; Özcan, M.; Pjetursson, B.E. Fracture strength of zirconia implant abutments on narrow diameter implants with internal and external implant abutment connections: A study on the titanium resin base concept. Clin. Oral Implant. Res. 2018, 29, 411-423. [CrossRef] 
27. Cao, Y.; Yu, C.; Wu, Y.; Li, L.; Li, C. Long-Term Survival and Peri-Implant Health of Titanium Implants with Zirconia Abutments: A Systematic Review and Meta-Analysis. J. Prosthodont. 2019, 28, 883-892. [CrossRef]

28. Fanali, S.; Tumedei, M.; Pignatelli, P.; Inchingolo, F.; Pennacchietti, P.; Pace, G.; Piattelli, A. Implant primary stability with an osteocondensation drilling protocol in different density polyurethane blocks. Comput. Methods Biomech. Biomed. Eng. 2020, 1-7. [CrossRef]

29. Fujiwara, S.; Kato, S.; Bengazi, F.; Urbizo Velez, J.; Tumedei, M.; Kotsu, M.; Botticelli, D. Healing at implants installed in osteotomies prepared either with a piezoelectric device or drills: An experimental study in dogs. Oral Maxillofac. Surg. 2020. [CrossRef]

30. Kermanshah, H.; Geramy, A.; Ebrahimi, S.F.; Bitaraf, T. IPS-Empress II inlay-retained fixed partial denture reinforced with zirconia bar: Three-dimensional finite element andin-vitrostudies. Acta Odontol. Scand. 2012, 70, 569-576. [CrossRef]

31. Kotsu, M.; Urbizo Velez, J.; Bengazi, F.; Tumedei, M.; Fujiwara, S.; Kato, S.; Botticelli, D. Healing at implants installed from $\sim 70$ - to $<10-\mathrm{Ncm}$ insertion torques: An experimental study in dogs. Oral Maxillofac. Surg. 2020. [CrossRef]

32. Scarano, A.; Di Carlo, F.; Piattelli, A. Effect of sterilization and cleansing on implantology drills: Zirconia vs steel. Ital. Oral Surg. 2008, 3, 61-72.

33. Scarano, A.; Valbonetti, L.; Marchetti, M.; Lorusso, F.; Ceccarelli, M. Soft Tissue Augmentation of the Face with Autologous Platelet-Derived Growth Factors and Tricalcium Phosphate. Microtomography Evaluation of Mice. J Craniofac. Surg. 2016, 27, 1212-1214. [CrossRef] [PubMed]

34. Scarano, A.; Piattelli, A.; Quaranta, A.; Lorusso, F. Bone Response to Two Dental Implants with Different Sandblasted/Acid-Etched Implant Surfaces: A Histological and Histomorphometrical Study in Rabbits. BioMed Res. Int. 2017, 2017, 8724951. [CrossRef] [PubMed]

35. Ibrahim, G.M.; Carter Snead, O.; Rutka, J.T.; Lozano, A.M. The most cited works in epilepsy: Trends in the "Citation Classics". Epilepsia 2012, 53, 765-770. [CrossRef]

36. Parker, J.N.; Lortie, C.; Allesina, S. Characterizing a scientific elite: The social characteristics of the most highly cited scientists in environmental science and ecology. Scientometrics 2010, 85, 129-143. [CrossRef]

37. Park, K.M.; Park, B.S.; Park, S.; Yoon, D.Y.; Bae, J.S. Top-100 cited articles on headache disorders: A bibliometric analysis. Clin. Neurol. Neurosurg. 2017, 157, 40-45. [CrossRef]

38. Lorusso, F.; Inchingolo, F.; Scarano, A. Scientific Production in Dentistry: The National Panorama through a Bibliometric Study of Italian Academies. BioMed Res. Int. 2020, 2020, 3468303. [CrossRef]

39. Jayaratne, Y.S.N.; Zwahlen, R.A. The evolution of dental journals from 2003 to 2012: A bibliometric analysis. PLOS ONE 2015, 10, e0119503. [CrossRef]

40. Pommer, B.; Valkova, V.; Ubaidha Maheen, C.; Fürhauser, L.; Rausch-Fan, X.; Seeman, R. Scientific Interests of 21st Century Clinical Oral Implant Research: Topical Trend Analysis. Clin. Implant Dent. Relat. Res. 2015, 18, 850-856. [CrossRef]

41. Gutiérrez-Vela, M.M.; Díaz-Haro, A.; Berbel-Salvador, S.; Lucero-Sánchez, A.; Robinson-García, N.; Cutando-Soriano, A. Bibliometric analysis of research on regenerative periodontal surgery during the last 30 years. J. Clin. Exp. Dent. 2012, 4, e112-e118. [CrossRef] [PubMed]

42. Tetè, S.; Zizzari, V.L.; De Carlo, A.; Lorusso, F.; Di Nicola, M.; Piattelli, A.; Gherlone, E.; Polimeni, A. Characterizing scientific production of Italian Oral Surgery professionals through evaluation of bibliometric indices. Ann. Di Stomatol. 2014, 5, 23. [CrossRef]

43. O'Brien, B.C.; Harris, I.B.; Beckman, T.J.; Reed, D.A.; Cook, D.A. Standards for Reporting Qualitative Research. Acad. Med. 2014, 89, 1245-1251. [CrossRef] [PubMed]

44. Liberati, A.; Altman, D.G.; Tetzlaff, J.; Mulrow, C.; Gøtzsche, P.C.; Ioannidis, J.P.A.; Clarke, M.; Devereaux, P.J.; Kleijnen, J.; Moher, D. The PRISMA statement for reporting systematic reviews and meta-analyses of studies that evaluate health care interventions: Explanation and elaboration. PLoS Med. 2009, 6, e1000100. [CrossRef]

45. Van Eck, N.J.; Waltman, L. Software survey: VOSviewer, a computer program for bibliometric mapping. Scientometrics 2010, 84, 523-538. [CrossRef]

46. Van Eck, N.J.; Waltman, L. VOSviewer manual. Leiden Univeristeit Leiden 2013, 1, 1-53.

47. Akça, K.; Cavusoglu, Y.; Uysal, S.; Cehreli, M.C. A Prospective, Open-Ended, Single-Cohort Clinical Trial on Early Loaded Titanium-Zirconia Alloy Implants in Partially Edentulous Patients: Up-to-24-Month Results. Int. J. Oral Maxillofac. Implant. 2013, 28, 573-578. [CrossRef] 
48. Balmer, M.; Spies, B.C.; Kohal, R.J.; Hämmerle, C.H.F.; Vach, K.; Jung, R.E. Zirconia implants restored with single crowns or fixed dental prostheses: 5-year results of a prospective cohort investigation. Clin. Oral Implant. Res. 2020, 31, 452-462. [CrossRef]

49. Balmer, M.; Spies, B.C.; Vach, K.; Kohal, R.-J.; Hämmerle, C.H.F.; Jung, R.E. Three-year analysis of zirconia implants used for single-tooth replacement and three-unit fixed dental prostheses: A prospective multicenter study. Clin. Oral Implant. Res. 2018, 29, 290-299. [CrossRef]

50. Becker, J.; John, G.; Becker, K.; Mainusch, S.; Diedrichs, G.; Schwarz, F. Clinical performance of two-piece zirconia implants in the posterior mandible and maxilla: A prospective cohort study over 2 years. Clin. Oral Implant. Res. 2015, 28, 29-35. [CrossRef]

51. Borgonovo, A.; Censi, R.; Dolci, M.; Vavassori, V.; Bianchi, A.; Maiorana, C. Use of endosseous one-piece yttrium-stabilized zirconia dental implants in premolar region: A two-year clinical preliminary report. Minerva. Stomatol. 2011, 60, 229-241. [PubMed]

52. Borgonovo, A.; Vavassori, V.; Censi, R.; Calvo, J.; Re, D. Behavior of endosseous one-piece yttrium stabilized zirconia dental implants placed in posterior areas. Minerva. Stomatol. 2013, 62, 247-257. [PubMed]

53. Bormann, K.-H.; Gellrich, N.-C.; Kniha, H.; Schild, S.; Weingart, D.; Gahlert, M. A prospective clinical study to evaluate the performance of zirconium dioxide dental implants in single-tooth edentulous area: 3-year follow-up. BMC Oral Health 2018, 18, 181. [CrossRef] [PubMed]

54. Cannizzaro, G.; Torchio, C.; Felice, P.; Leone, M.; Esposito, M. Immediate occlusal versus non-occlusal loading of single zirconia implants. A multicentre pragmatic randomised clinical trial. Eur. J. Oral Implant. 2010, 3, 111-120.

55. Cionca, N.; Müller, N.; Mombelli, A. Two-piece zirconia implants supporting all-ceramic crowns: A prospective clinical study. Clin. Oral Implant. Res. 2015, 26, 413-418. [CrossRef]

56. Jung, R.E.; Grohmann, P.; Sailer, I.; Steinhart, Y.-N.; Fehér, A.; Hämmerle, C.; Strub, J.R.; Kohal, R. Evaluation of a one-piece ceramic implant used for single-tooth replacement and three-unit fixed partial dentures: A prospective cohort clinical trial. Clin. Oral Implant. Res. 2015, 27, 751-761. [CrossRef]

57. Kniha, K.; Kniha, H.; Möhlhenrich, S.C.; Milz, S.; Hölzle, F.; Modabber, A. Papilla and alveolar crest levels in immediate versus delayed single-tooth zirconia implants. Int. J. Oral Maxillofac. Surg. 2017, 46, 1039-1044. [CrossRef]

58. Kniha, K.; Schlegel, K.A.; Kniha, H.; Modabber, A.; Hölzle, F.; Kniha, K. Evaluation of peri-implant bone levels and soft tissue dimensions around zirconia implants-A three-year follow-up study. Int. J. Oral Maxillofac. Surg. 2017, 47, 492-498. [CrossRef]

59. Kohal, R.-J.; Patzelt, S.B.M.; Butz, F.; Sahlin, H. One-piece zirconia oral implants: One-year results from a prospective case series. 2. Three-unit fixed dental prosthesis (FDP) reconstruction. J. Clin. Periodontol. 2013, 40, 553-562. [CrossRef] [PubMed]

60. Kohal, R.-J.; Spies, B.C.; Bauer, A.; Butz, F. One-piece zirconia oral implants for single-tooth replacement: Three-year results from a long-term prospective cohort study. J. Clin. Periodontol. 2018, 45, 114-124. [CrossRef] [PubMed]

61. Koller, M.; Steyer, E.; Theisen, K.; Stagnell, S.; Jakse, N.; Payer, M. Two-piece zirconia versus titanium implants after 80 months: Clinical outcomes from a prospective randomized pilot trial. Clin. Oral Implant. Res. 2020, 31, 388-396. [CrossRef] [PubMed]

62. Lorenz, J.; Giulini, N.; Hölscher, W.; Schwiertz, A.; Schwarz, F.; Sader, R. Prospective controlled clinical study investigating long-term clinical parameters, patient satisfaction, and microbial contamination of zirconia implants. Clin. Implant Dent. Relat. Res. 2019, 21, 263-271. [CrossRef] [PubMed]

63. Osman, R.B.; Payne, A.; Duncan, W.; Ma, S. Zirconia implants supporting overdentures: A pilot study with novel prosthodontic designs. Int. J. Prosthodont. 2013, 26, 277-281. [CrossRef]

64. Osman, R.B.; Swain, M.V.; Atieh, M.; Ma, S.; Duncan, W. Ceramic implants (Y-TZP): Are they a viable alternative to titanium implants for the support of overdentures? A randomized clinical trial. Clin. Oral Implant. Res. 2014, 25, 1366-1377. [CrossRef] [PubMed]

65. Patankar, A.; Kshirsagar, R.; Patankar, S.; Pawar, S. Immediate, non submerged root analog zirconia implant in single rooted tooth replacement: Case report with 2 years follow up. J. Maxillofac. Oral Surg. 2016, 15, 270-273. [CrossRef] 
66. Payer, M.; Arnetzl, V.; Kirmeier, R.; Koller, M.; Arnetzl, G.; Jakse, N. Immediate provisional restoration of single-piece zirconia implants: A prospective case series-results after 24 months of clinical function. Clin. Oral Implant. Res. 2012, 24, 569-575. [CrossRef]

67. Payer, M.; Heschl, A.; Koller, M.; Arnetzl, G.; Lorenzoni, M.; Jakse, N. All-ceramic restoration of zirconia two-piece implants-A randomized controlled clinical trial. Clin. Oral Implant. Res. 2015, 26, 371-376. [CrossRef]

68. Pirker, W.; Kocher, A. Immediate, non-submerged, root-analogue zirconia implant in single tooth replacement. Int. J. Oral Maxillofac. Surg. 2008, 37, 293-295. [CrossRef]

69. Pirker, W.; Kocher, A. Immediate, non-submerged, root-analogue zirconia implants placed into single-rooted extraction sockets: 2-year follow-up of a clinical study. Int. J. Oral Maxillofac. Surg. 2009, 38, 1127-1132. [CrossRef]

70. Siddiqi, A.; Kieser, J.A.; De Silva, R.K.; Thomson, W.M.; Duncan, W.J. Soft and Hard Tissue Response to Zirconia versus Titanium One-Piece Implants Placed in Alveolar and Palatal Sites: A Randomized Control Trial. Clin. Implant Dent. Relat. Res. 2015, 17, 483-496. [CrossRef]

71. Spies, B.C.; Balmer, M.; Jung, R.E.; Sailer, I.; Vach, K.; Kohal, R.-J. All-ceramic, bi-layered crowns supported by zirconia implants: Three-year results of a prospective multicenter study. J. Dent. 2017, 67, 58-65. [CrossRef] [PubMed]

72. Spies, B.C.; Balmer, M.; Jung, R.E.; Sailer, I.; Vach, K.; Kohal, R.J. All-ceramic single crowns supported by zirconia implants: 5-year results of a prospective multicenter study. Clin. Oral Implant. Res. 2019, 30, 466-475. [CrossRef] [PubMed]

73. Spies, B.C.; Balmer, M.; Patzelt, S.B.M.; Vach, K.; Kohal, R.J. Clinical and Patient-reported Outcomes of a Zirconia Oral Implant: Three year Results of a Prospective Cohort Investigation. J. Dent. Res. 2015, 94, 1385-1391. [CrossRef] [PubMed]

74. Spies, B.C.; Sperlich, M.; Fleiner, J.; Stampf, S.; Kohal, R.-J. Alumina reinforced zirconia implants: 1-year results from a prospective cohort investigation. Clin. Oral Implant. Res. 2016, 27, 481-490. [CrossRef] [PubMed]

75. Spies, B.C.; Witkowski, S.; Vach, K.; Kohal, R.J. Clinical and patient-reported outcomes of zirconia-based implant fixed dental prostheses: Results of a prospective case series 5 years after implant placement. Clin. Oral Implant. Res. 2017, 29, 91-99. [CrossRef] [PubMed]

76. Borgonovo, A.-E.; Fabbri, A.; Vavassori, V.; Censi, R.; Maiorana, C. Multiple teeth replacement with endosseous one-piece yttrium-stabilized zirconia dental implants. Med. Oral Patol. Oral Cir. Bucal. 2012, 17, e981-e987. [CrossRef] [PubMed]

77. Greenhalgh, T. How to read a paper: Getting your bearings (deciding what the paper is about). BMJ 1997, 315, 243-246. [CrossRef]

78. Guyatt, G.H.; Sackett, D.L.; Sinclair, J.C.; Hayward, R.; Cook, D.J.; Cook, R.J.; Bass, E.; Gerstein, H.; Haynes, B.; Holbrook, A. Users' guides to the medical literature: IX. A method for grading health care recommendations. JAMA 1995, 274, 1800-1804. [CrossRef]

79. Scarano, A.; Crocetta, E.; Quaranta, A.; Lorusso, F. Influence of the thermal treatment to address a better osseointegration of Ti6Al4V dental implants: Histological and histomorphometrical study in a rabbit model. BioMed Res. Int. 2018. [CrossRef]

80. Scarano, A.; Degidi, M.; Perrotti, V.; Degidi, D.; Piattelli, A.; Iezzi, G. Experimental Evaluation in Rabbits of the Effects of Thread Concavities in Bone Formation with Different Titanium Implant Surfaces. Clin. Implant Dent. Relat. Res. 2013, 16, 572-581. [CrossRef]

81. Van Eck, N.J.; Waltman, L. Accuracy of citation data in Web of Science and Scopus. arXiv 2019, arXiv:1906.07011.

82. Feijoo, J.F.; Limeres, J.; Fernández-Varela, M.; Ramos, I.; Diz, P. The 100 most cited articles in dentistry. Clin. Oral Investig. 2014, 18, 699-706. [CrossRef] [PubMed]

83. Ugolini, D.; Neri, M.; Cesario, A.; Bonassi, S.; Milazzo, D.; Bennati, L.; Lapenna, L.M.; Pasqualetti, P. Scientific production in cancer rehabilitation grows higher: A bibliometric analysis. Supportive Care Cancer 2012, 20, 1629-1638. [CrossRef] [PubMed]

84. Davis, P.M. Open access, readership, citations: A randomized controlled trial of scientific journal publishing. FASEB J. 2011, 25, 2129-2134. [CrossRef] [PubMed] 
85. Gargouri, Y.; Hajjem, C.; Larivière, V.; Gingras, Y.; Carr, L.; Brody, T.; Harnad, S. Self-selected or mandated, open access increases citation impact for higher quality research. PLoS ONE 2010, 5, e13636. [CrossRef] [PubMed]

86. Hua, F.; Shen, C.; Walsh, T.; Glenny, A.-M.; Worthington, H. Open Access: Concepts, findings, and recommendations for stakeholders in dentistry. J. Dent. 2017, 64, 13-22. [CrossRef] [PubMed]

Publisher's Note: MDPI stays neutral with regard to jurisdictional claims in published maps and institutional affiliations.

(C) 2020 by the authors. Licensee MDPI, Basel, Switzerland. This article is an open access article distributed under the terms and conditions of the Creative Commons Attribution (CC BY) license (http://creativecommons.org/licenses/by/4.0/). 